\title{
Poststroke Recovery
}

\author{
Emerging Complementary Therapies
}

\section{Jane Hart, MD}

Stroke is the third leading cause of death in the United States, and, each year, nearly 800,000 Americans experience a new or recurrent stroke. ${ }^{1}$ Stroke is also a leading cause of disability in the United States. People who experience stroke may struggle with significant physical, psychologic, and social challenges. For example, stroke survivors may be left with significant disabilities including inability to use their limbs or impaired ability to communicate verbally or understand others. Even minimal disability can affect people's lives significantly by, for example, prohibiting them from driving or from carrying out activities of daily living.

The good news is that, in the early stages of stroke recovery, the brain may undergo significant plastic changes, which, as one reviewer notes, may be enhanced by multimodal environmental stimulation. ${ }^{3}$ This is an opportune time to engage a patient who is recovering from a stroke in both conventional and complementary therapies that may optimize his or her motor and cerebral functioning. Emerging research suggests a role for complementary therapies for improving functionality and quality of life (QoL) in the stroke recovery phase.

\section{Background}

Stroke is caused either by (1) obstruction of a blood vessel (ischemic stroke) from thrombi or emboli or by (2) bleeding from a blood vessel (hemorrhagic stroke). The majority of strokes are ischemic in nature.

Major warning signs of stroke include: sudden headache; sensory impairment such as a change in vision; difficulty with speaking or difficulty with understanding someone else; and/ or difficulty with walking or using a limb. A transient ischemic attack or TIA is a sign of impending stroke, in which similar symptoms as a stroke may occur but are only temporary.

A stroke is an emergency and needs immediate attention, because early intervention may help prevent or limit long-term disability. For patients who have ischemic stroke, for instance, tissue plasminogen activator (tPA), if administered in the early phase, may break up a clot, save the patient's life, and help prevent long-term disability.
Who gets strokes? A person may experience a stroke at any age, but strokes are more common in people over 65 years of age. ${ }^{4}$ African Americans have nearly twice the risk of a first stroke compared with whites. ${ }^{2}$ Risk factors for stroke include prior history of a stroke, high blood pressure (BP), atherosclerosis, atrial fibrillation, high cholesterol, diabetes, smoking, and family history of stroke. ${ }^{5}$

Important measures for preventing stroke include control of $\mathrm{BP}$, treating dyslipidemias, managing diabetes, treating known atherosclerosis or preventing atherosclerosis, and smoking cessation. Prevention is essential because disability following stroke can be significant and lifelong.

\section{Patterns of CAM Usage}

Similar to a person who experiences a serious cancer, a person who has a stroke may experience a significant physical, emotional, and financial toll. Poststroke, a person may have motor deficits, cognitive deficits, financial burden, job loss, fractured relationships, sleep disorders, fatigue, and significant grief. While challenged with these and other issues, a person may turn to complementary therapies for relief. An integrated stroke rehabilitation program may utilize acupuncture, acupressure, exercise, dietary interventions, mindfulness and other mind-body relaxation and stress-reducing practices, social support, biofeedback, massage, and others kinds of complementary and alternative medicine (CAM) approaches. ${ }^{6}$

According to one review, results from the 2002 National Health Survey reveal that nearly half of stroke survivors use some type of CAM. ${ }^{7}$ Acupuncture was used more frequently among stroke survivors, compared with people with other conditions, and CAM was used more frequently among patients who reported delays in seeking medical care because of cost and among those with recent neck pain. CAM was used less frequently among patients older than age 65, non-Hispanic blacks, and patients who did not graduate from high school.

In a Korean study, among 304 patients who had stroke, 54\% had used CAM and 57\% of users felt CAM was effective; $84 \%$ believed CAM had eased stroke symptoms; and 16\% felt it 
was effective in helping achieve relaxation. ${ }^{8}$ In this study, $92 \%$ used Oriental medical treatments, $36 \%$ used over-the-counter (OTC) plant- or animal-derived products, $24 \%$ used minerals and vitamins, and $11 \%$ used manual therapies.

\section{Research}

People poststroke may seek CAM therapies for a wide variety of physical symptoms, such as pain or immobility, and for relief from psychologic symptoms such as grief, depression, or anxiety. The fact that people in the stroke recovery phase experience significant diversity in their sequelae and symptomatology makes evaluating the available body of literature on CAM and stroke a challenge, because outcomes are not always well-defined.

One review notes that health-related quality of life (HRQoL) is a significant outcome to follow for patients who experience stroke. ${ }^{9}$ The review notes that poststroke depression affects HRQoL, functional recovery, cognitive function, and health care use in survivors of stroke. Factors that suggest greater risk for adverse outcomes include advancing age, anxiety in patients or caregivers, high dependency, and poor family support. The review concludes that physical and psychosocial well-being are greatly affected in survivors of stroke and their caregivers.

Another study examining important HRQoL issues after stroke surveyed 188 people poststroke through a variety of measurement tools 2-3 weeks after discharge. ${ }^{10}$ Depressive symptoms were associated with lower HRQoL. Fewer depressive symptoms, and higher participation in social activities (such as outdoor activities) and engaging in personal interests were related to good HRQoL.

Various complementary modalities have been found to be beneficial in stroke recovery. Research continues to emerge in this area.

\section{Acupuncture}

Acupuncture may benefit survivors of stroke in many ways. A large number of studies have examined the benefits of acupuncture in stroke recovery, and results are mixed. A Cochrane review on acupuncture for stroke rehabilitation states that there is no clear evidence of the effects of acupuncture on stroke rehabilitation, but that acupuncture has biologic effects that might improve stroke recovery or rehabilitation. The reviewers examined five randomized trials and note that the studies were of poor quality and that no definite conclusions could be drawn. These reviewers recommend large, randomized controlled trials (RCTs) for further investigation. ${ }^{11}$

Another review of acupuncture for addressing acute stroke yielded similar results, and the reviewers state that there is no clear evidence of benefit from acupuncture for patients with acute stroke with respect to reducing risk of death, or help with everyday activities. These reviewers also recommend larger randomized trials to assess the benefits and safety issues of acupuncture in acute stroke care. ${ }^{12}$
However, a 2010 systematic review and meta-analysis of randomized trials, published in Stroke, on acupuncture use in poststroke rehabilitation concludes that RCTs demonstrate that acupuncture may be effective for poststroke rehabilitation. This review covered 35 articles written in Chinese and 21 written in English; 80\% of the studies reported a significant benefit from acupuncture.

Another group of researchers note wisely that "the effectiveness of acupuncture as an adjunct to standard post-stroke rehabilitation programs may be demonstrated when more specific measures of stroke motor recovery and physical function are used." ${ }^{14}$ This study examined the effect of acupuncture on motor recovery and physical function. The researchers randomized 32 patients with acute stroke to standard rehabilitation or acupuncture plus standard rehabilitation. Motor recovery was measured by the Fugl-Meyer (FM) Assessment, and physical function was measured by Functional Independence Measurement (FIM). While there was no difference in total FM or FIM between the two groups, statistically significant benefit in the acupuncture group was seen for the FM lower-extremity motor-function subscale and the tub/transfer mobility subscale of the FIM.

It is important to realize that the topic of "stroke recovery and acupuncture" is very broad. There are a number of ways that acupuncture may be applied in stroke survivors. Acupuncture may be used to improve motor or cognitive function; to

\section{Clearly, there are individuals poststroke who benefit significantly from acupuncture for a wide variety of conditions.}

manage pain; or to address insomnia, anxiety, depression, etc. Clearly, there are individuals poststroke who benefit significantly from acupuncture for a wide variety of conditions. It is therefore essential that research studies evaluating acupuncture and "stroke rehabilitation" define endpoints clearly and report on specific interventions that were used to treat specific conditions and achieve specific outcomes.

\section{Motor Imagery}

Motor imagery (MI), along with conventional physical therapy (PT) or occupational therapy (OT), may improve motor functionality better than conventional treatments alone. Motor imagery is a process whereby participants visualize a specific action without actually making the movement. A popular area in which this kind of therapy has proven benefit is athletes' use of such imagery to enhance performance.

One systematic review explored four randomized controlled trials that analyzed the effect of MI plus conventional therapy, compared with the effect of conventional therapy alone, in patients who had experienced cortical stroke. ${ }^{15}$ An example of how 
MI was used by study participants involved visualizing tasks to be accomplished through mental imagery and visualizing problems and solutions to any obstacles in performing the task. There was a significantly higher level of performance for tasks for which the participants were trained as well as tasks for which participants were untrained in the imagery group, compared with the control group. In this particular study, OTs provided the MI training, which was delivered to the intervention group in 60-minute sessions, 5 days a week, for 3 weeks, along with 60-minute physical therapy sessions, 5 days a week for 3 weeks.

The reviewers note that MI may be used during all phases of stroke recovery and, in the early phase, MI may be used to help patients visualize carrying out a task that they may not physically be able to carry out because of motor impairment. The reviewers conclude that "motor imagery provides additional benefits to conventional physiotherapy or occupational therapy." These reviewers recommend larger and high-quality studies to determine the benefits of MI and the appropriate frequency and timing of such interventions. "MI appears to be an attractive treatment option, easy to learn and to apply, and the intervention is neither physically exhausting nor harmful. Therefore, the authors believe that MI may generate additional benefit for patients," the reviewers comment.

\section{Music Therapy}

Music therapy has been shown to improve cognitive function, motor function, and mood in people with various disorders. One study tested whether everyday music listening could facilitate recovery of cognitive function and mood after middle-cerebral-artery stroke (MCA). ${ }^{3}$ In this study, 60 people with right- or left-hemisphere MCA were randomly assigned to a music group, a language group, or a control group. For 2 months, the music and language groups listened daily to selfselected music or audiobooks and the control group received no listening material. All participants received conventional care and rehabilitation.

Participants underwent extensive neuropsychologic assessment at baseline, 3 months, and 6 months. Fifty-four participants completed the study. The music group experienced greater recovery in verbal memory and focused attention, compared with the language and control groups, and experienced less depressed and confused mood, compared with the control group. The researchers conclude that music listening during the early poststroke state may enhance cognitive recovery and prevent negative mood.

\section{Art Therapy}

Art therapy may help meet the cognitive, physical, and emotional needs of patients who survive stroke. A report on art therapy in stroke rehabilitation notes that art therapy may be particularly suited for people with: psychologic distress who are unable to use verbal forms of psychotherapy or counseling; emotional or psychosocial challenges that are resistant to conventional rehabilitation; and specific emotional issues related to "living with a stroke," such as grief, shame, helplessness, anger, depression, loss, adjustment and coping. ${ }^{16}$
According to one case study, art therapy may be used by people with brain injury to convey emotions, ease feelings of helplessness, master anxiety, work through grief, and adapt to change. ${ }^{17}$ Survivors of stroke in one rehabilitation setting reported that art therapy helped them feel more in control, more relaxed, more sociable, more worthwhile, and more interested in things.

\section{Stroke Support Groups}

Stroke support groups may help improve QoL, and ease depression and anxiety. Such groups may also be instrumental in educating participants and caregivers about important stroke knowledge such as stroke warning signs, risk factors, and preventive measures. This kind of education is imperative, because according to the National Stroke Association, 5\%-14\% of people who experience a stroke will have another stroke within 1 year, and $24 \%$ of women and $42 \%$ of men will have a recurrent stroke within 5 years. ${ }^{18}$

One study examining stroke knowledge among supportgroup members surveyed 133 stroke support group attendees, among which 70\% had experienced stroke (other participants were family members or volunteers). ${ }^{19}$ In this study, $80 \%$ of participants had good symptom knowledge (knew at least two stroke symptoms); 65\% had good riskfactor knowledge (knew at least two stroke risk factors); and $80 \%$ had good action knowledge (knew that immediate hospital admission or an emergency call was necessary in case of stroke). The researchers comment: "The high information status demonstrated among these laypersons stands out markedly compared with stroke knowledge of the general population," and conclude that "support groups should be viewed as important partners in community stroke education."

\section{Conclusion}

One of the challenges in CAM research is to define endpoints, interventions, and outcomes clearly within specific populations. Stroke is a condition that leaves individuals with a wide variety of physical and emotional challenges and, therefore, one cannot really look at "stroke" and CAM in general. Instead, it is essential to look at the conditions that stroke survivors experience, such as grief, depression, insomnia, and pain, and see what modalities are proven most useful for those conditions.

It is also essential that assessment of a survivor's physical and emotional needs is performed early in the stroke-recovery phase to connect that person with conventional and complementary therapies that will strengthen, improve, and optimize that person's recovery course.

\section{References}

1. American Stroke Association. Impact of Stroke. Online document at: www. strokeassociation.org/presenter.jhtml?identifier=1033 Accessed July 15, 2010. 
2. American Heart Association. Stroke: Heart Disease and Stroke Statistics. 2010 Update at-a-Glance. Online document at: www.americanheart.org/ downloadable/heart/1265665152970DS3241\%20HeartStrokeUpdate_2010. pdf Accessed August 3, 2010

3. Sarkamo T, Tervaniemi M, Laitinen S, et al. Music listening enhances cognitive recovery and mood after middle cerebral artery stroke. Brain 2008;131:866-876.

4. Internet Stroke Center at Washington University. Stroke Statistics. Online document at: www.strokecenter.org/patients/stats.htm Accessed August 3, 2010.

5. National Stroke Association. Stroke Risk Factors. Am I at Risk for a Stroke? Online document at: www.stroke.org/site/PageServer?pagename=RISK Accessed August 3, 2010.

6. Magnusson G, Ballegaard S, Karpatschof B, Nyboe J. Long-term effects of integrated rehabilitation in patients with stroke: A nonrandomized comparative feasibility study. J Altern Complement Med 2010;16:369-374.

7. Shah SH, Englehardt R, Ovbiagele B. Patterns of complementary and alternative medicine use among United States stroke survivors. J Neurol Sci 2008;271:180-185.

8. Shin Y-I, Yang C-Y, Joo M-C, et al. Patterns of using complementary and alternative medicine by stroke patients at two university hospitals in Korea.eCAM 2008;5:231-235.

9. Carod-Artal FJ, Egido JA. Quality of life after stroke: The importance of a good recovery. Cerebrovasc Dis 2009;27(suppl1):204-214.

10. Almborg AH,Ulander K, Thulin A,Berg S.Discharged after stroke-important factors for health-related quality of life. J Clin Nurs 2010;19:2196-2206.

11. Wu HM, Tang J-L, Lin XP, et al. Acupuncture for stroke rehabilitation. Cochrane Database Syst Rev 2006;3:CD004131.

12. Zhang S, Liu M, Asplund K, Li L. Acupuncture for acute stroke. Cochrane Database Syst Rev 2005;2:CD003317.
13. Wu P, Mills E, Moher D, Seely D. Acupuncture in poststroke rehabilitation: A systematic review and meta-analysis of randomized trials, Stroke 2010;41:e71-e179.

14. Alexander DN, Cen S, Sullivan KJ, et al. Effects of acupuncture treatment on poststroke motor recovery and physical function: A pilot study. Neurorehabil Neural Repair 2004;18:259-267.

15. Zimmermann-Schlatter A, Schuster C, Puhan MA, et al. Efficacy of motor imagery in post-stroke rehabilitation: A systematic review. J Neuroeng Rehabil 2008;5:8.

16. British Association of Art Therapists. Art Therapies in the Stroke Pathway. Online document at: www.baat.org/ARTSTHERAPIESANDSTROKE07. pdf Accessed August 9, 2010.

17. British Association of Art Therapists. Case Study. Online document at: www.baat.org/CaseStudyStroke.pdf Accessed August 9, 2010.

18. National Stroke Association. Secondary (Recurrent) Risk. Online document at: www.stroke.org/site/PageServer?pagename=RECUR Accessed August 3, 2010.

19. Weltermann BM, Homann J, Rogalewski A, et al. Stroke knowledge among stroke support group members. Stroke 2000;31:1230-1233.

Jane Hart, MD, is a clinical instructor in internal medicine and chair of the Integrative Medicine Committee at Case Western Reserve University School of Medicine, in Cleveland, Ohio.

To order reprints of this article, e-mail Karen Ballen at: Kballen@liebertpub.com or call (914) 740-2100. 